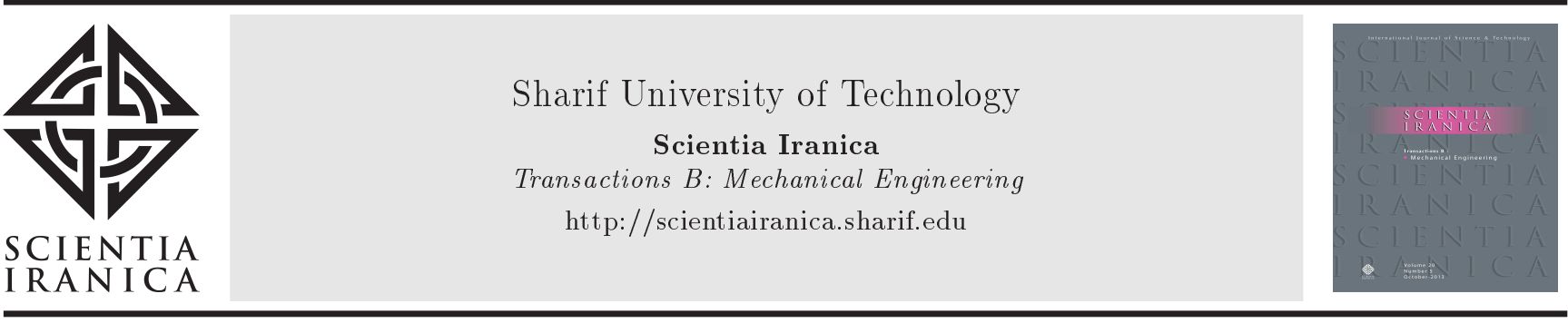

\title{
Numerical simulation of turbulent oscillating flow in porous media
}

\author{
A. Kardgar ${ }^{\mathrm{a}, \mathrm{b}}$ and A. Jafarian ${ }^{\mathrm{a}, *}$ \\ a. Department of Mechanical Engineering, Tarbiat Modares University, Tehran, P.O. Box 14115-143, Iran. \\ b. Department of Mechanical Engineering, University of Mazandaran, Babolsar, P.O. Box 47416-13534, Iran. \\ Received 13 January 2019; received in revised form 12 December 2019; accepted 18 January 2020
}

\section{KEYWORDS}

Oscillating flow;

Turbulence;

Porous media;

$k-\varepsilon$ model;

Volume averaging.

\begin{abstract}
Two macroscopic turbulence models, namely P-dL and N-K, have been proposed in recent years to simulate turbulent unidirectional flow in porous media. In this paper, a modification to the N-K model was proposed for turbulent oscillating flow in porous media. To this end, the flow in porous media was simulated on a microscale using a periodic array. The $k-\varepsilon$ model was employed to solve turbulent oscillating flow in periodic array. A control volume approach was also used to discretize Navier-Stokes equations and $k-\varepsilon$ equations and the well-established SIMPLE method was applied to investigate pressure and velocity coupling. In order to modify the N-K model, the effects of different parameters such as frequency and Reynolds number were studied and the constants in the source terms of turbulent kinetic energy and its dissipation rate were modified in comparison to Re according to the microscale results. To validate the new modified constants, a modified N-K model was applied to turbulent oscillating flow in porous media and the obtained results were compared to the original N-K macroscopic model.
\end{abstract}

(C) 2021 Sharif University of Technology. All rights reserved.

\section{Introduction}

Porous media and the corresponding heat transfer phenomenon have recently drawn considerable attention due to their relevance to a wide range of applications in science and engineering such as underground heat exchangers for energy saving, solar collectors, geothermal energy, oil extraction, cooling electronic devices, nuclear reactors, thermal insulations, etc. [1]. Due to different applications of porous media in different industries, the length scale of a pore in the porous media can range from Angstrom to centimeters or even

*. Corresponding author. Tel.: +982182884909 E-mail addresses: a.kardgar@umz.ac.ir (A. Kardgar); jafarian@modares.ac.ir. (A. Jafarian)

doi: $10.24200 /$ sci. 2020.52521 .2788 higher. Therefore, in these types of flows, the porescale Reynolds number $\left(\operatorname{Re}_{p}=\rho_{f} u_{D} d_{p} / \mu\right)$ is inclusive of a wide range [2]. Jolls and Hanratty [3], Dybbs and Edwards [4], and Horton and Pokrajac [5] conducted several studies to confirm the existence of turbulence in the porous media. Jolls and Hanratty mentioned that transition from laminar to turbulent flow occurred at Reynolds numbers 110 to 150 . Using their experimental studies, Dybbs and Edwards showed that fluid flow could reveal some characteristics of turbulence in Reynolds number slightly above a few hundreds. They emphasized the existence of four flow regimes including Darcy regime $\left(\operatorname{Re}_{p}<1\right)$, Forchheimer regime $(1-10<$ $\left.\operatorname{Re}_{p}<150\right)$, transient Laminar $\left(150<\operatorname{Re}_{p}<300\right)$, and turbulent regime $\left(300<\operatorname{Re}_{p}\right)$. Since transition to the turbulent flow in the porous media occurs at low Reynolds numbers, flow is in the turbulent form in many different applications of the porous media. Due 
to the inherent difficulty of measuring flow velocity and turbulence intensity on a microscale, macroscale modeling of the turbulence was used to investigate it in porous media. To model a turbulent flow in the mentioned media, researchers have employed the same volume averaging method as that used in the laminar flow. In this regard, time averaging is used for handling turbulence and volume averaging to simultaneously model the flow in the porous media. Different models vary in terms of time order and volume averaging. However, with respect to the definition of averaging, different definitions can be provided for turbulence kinetic energy and its dissipation rate [6]. Almost all models are based on the $k-\varepsilon$ model in the non-porous flow.

Masuoka and Takatsu proposed a zero-equation turbulence model for flow in the porous media in which eddy viscosity was a function of permeability, velocity (turbulent kinetic energy) of the fluid inside the media, and Forchheimer coefficient. They argued that momentum transfer of turbulent flow in the porous media was conducted in two types of vortexes: pseudo and void vortexes. Therefore, the viscosity of turbulent flow is the result of adding both pseudo and void vortexes together $\mu_{t}=\mu_{t, \text { pseudo }}+\mu_{t, \text { void }}$. Figure 1 shows both pseudo and void vortexes [7].

The model proposed by Masuoka and Takatsu applies transient conditions to the turbulent flow after Darcy regime; however, in the media, Forchheimer regime apparently exists after the Darcy regime and the transient condition cannot be initiated right after the mentioned regime. The above authors considered $\operatorname{Re}_{p}=10$ as the outset of the turbulent flow. They also correlated Forchheimer flow resistance with turbulent flow characteristics, an assumption that none of the references have verified. Therefore, their model is based on an incorrect assumption and the obtained results

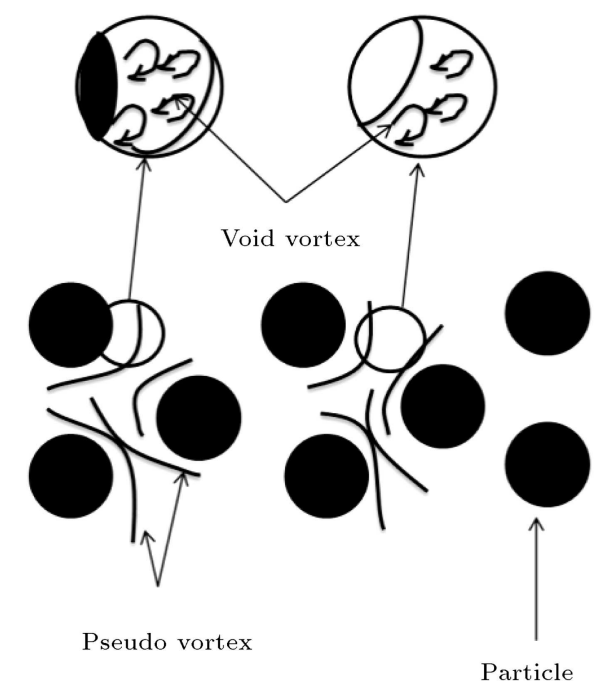

Figure 1. Schematics of void and pseudo vortexes in the packed bed [7]. cannot be trustworthy [8]. Alvarez et al. proposed an equation to model the turbulent flow in a packed sphere bed. They calculated the constants from experimental results. However, the constants they calculated can only be used in certain conditions and geometries [9].

The $k-\varepsilon$ model was specifically designed for planar shear layers and recirculating flows. This model is the most widely used and validated turbulence model with applications ranging from industrial to environmental flows. Models proposed by a number of researchers are based on $k-\varepsilon$ turbulence model. Antohe and Lage used time averaging of volumeaveraged Navier-Stokes equations to provide a twoequation model for turbulent flow in porous media [10]. The kinetic energy in the equations they suggested was considered zero for a unidirectional fully-developed steady flow. Nakayama and Kuwahara presented a two-equation model using volume averaging of timeaveraged equations [11]. Pedras and de Lemos showed that the order of averaging Navier-Stokes equation had no effect on the results, except for the turbulence kinetic energy equation [12]. They also added turbulence on a pore scale to the model using volume averaging after time averaging. Finally, they proposed an equation to model the terms associated with the turbulence kinetic energy equation and its dissipation rate after volume averaging [13]. However, it should be noted that the presented source terms are not valid for all porous media. Despite the difficulties involved, the constants of N-K and P-dL terms should be determined based on experimental data. To this end, a periodic array was used to obtain the constants of the proposed equations. Guo et al. [14] showed that the proposed PdL model had a number of deficiencies. They concluded that Eddy viscosity was highly dependent on the pore size in the P-dL model due to the presence of $k / u_{D}^{2}$ term in the Eddy viscosity of P-dL model (Eq. (2)). In case of a fully developed flow, another deficiency of the P-dL model lies in the source terms so that both equations of this model would turn into one. In fact, all terms of the transport equation except the source term are omitted.

$$
\begin{aligned}
& \epsilon=\frac{c_{k} k \varphi u}{\sqrt{K}}, \\
& \mu_{t \varphi}=\frac{c_{\mu} \rho_{f} u_{D} d_{p} \varphi^{3 / 2}}{c_{k} \sqrt{150}(1-\varphi)} \frac{k}{u_{D}^{2}} .
\end{aligned}
$$

Therefore, the amount of Eddy viscosity in the P-dL model is dependent on the dimensionless turbulence energy $\left(\frac{k}{u_{D}^{2}}\right)$, which is subject to turbulence intensity at the entrance of the porous media. The obtained amount is inconsistent with the previous results in terms of the independency of the values of turbulence in the developed area of the porous media in inlet conditions. Guo et al. compared three different models 
for flow simulation in a porous medium and concluded that Nakayam and Kuwahara model was superior to others and could provide more realistic results [14]. Nakayama and Kuwahara suggested that dimensionless turbulent energy for $\operatorname{Re}_{p}>3000$ was independent of the Reynolds number and that the N-K model benefited from values independent of $\operatorname{Re}_{p}$ used for calculating $G_{e}^{i}$ and $G_{k}^{i}$. The mentioned model has been extensively used for different turbulence models in porous media like flow in channels, pipes, packed beds, etc. in recent years. Nouri-Borujerdi and SeyyedHashemi studied heat transfer in the turbulent flow in porous media. They used $k-\varepsilon$ model to handle turbulence in porous media and investigate the effects of different porous thicknesses and Darcy numbers on the pressure drop [15]. Kazerooni and Hannani simulated turbulent flow in porous media using v2f model and finite element method. They concluded that since the inherent advantages of using a v2f model were not clearly identified by macroscopic models, they could be mathematically proved [16].

Kim and Kang conducted a numerical simulation to study anomalous transport through free-flowporous media interface [17]. They suggested that RANS models could efficiently be applied to simulate turbulent flow in porous media on a pore scale. De Lemos and Assato investigated turbulence structure and heat transfer in a sudden expansion with a porous insert using linear and non-linear turbulence models. Their results revealed complete damping of turbulence kinetic energy generation along the channel for thick porous inserts [18]. Soulaine and Quintard proposed an approach to derive a macroscale momentum equation, which was free from the turbulence model, chosen for the pore-scale simulations and it was able to capture large-scale anisotropy. This technique offers a macroscale generalized Darcy-Forchheimer equation which is associated with a closure problem that can be used to evaluate the apparent permeability tensor including inertia effects [19]. Torabi et al. analyzed fluid flow, heat transfer, and entropy generation of turbulent forced convection in isotropic porous media using RANS models [20]. They investigated different Re numbers, porosities, and cross-sections for two turbulence models. Having considered symmetric contours of thermophysical properties, they concluded that the RNG $k-\varepsilon$ model presented a more successful illustration of contours than the SST k- $\omega$ model. Kundu et al. investigated the turbulent flow in isotropic porous media numerically. They compared v2f model and low Re $-k-\varepsilon$-Lam-Bremhorst (LB) with Large Eddy Simulation (LES) predictions and found that the results of low $\operatorname{Re}-k-\varepsilon$-(LB) were closer to those of LES [21]. Chu et al. conducted direct numerical simulation to study heat transfer and pressure drop in the porous media. To this end, they used a periodic square cylinder in a staggered array and concluded that increasing Reynolds numbers led to greater pressure loss rather than improved heat transfer [22]. Four types of two-equation turbulence models were compared while simulating the porous media of Diesel Particulate Filter (DPF) and swirltype regenerator burner. It was observed that backflow features of the realizable $k-\varepsilon$ were clearer than those of RNG $k-\varepsilon$ model [23]. Khan and Straatman proposed two equation turbulence models to investigate non-equilibrium heat transfer for unidirectional flow in the porous media [24]. Due to the complexity of turbulent flow in the porous media, Linsong et al. studied turbulent flow in randomly packed beds using pore-scale three-dimensional simulation. They pointed to the existence of complicated turbulent eddy structures in the pores of the packed bed [25].

Despite much academic effort to propose an accurate model for simulating turbulence in porous media, macroscopic models developed so far are unsuitable for simulating unsteady and oscillating flows. In this respect, the present study aimed to provide a model better suited for simulating unsteady oscillating flow by correcting the coefficients of the N-K model. To achieve this objective, the flow was first simulated in porous media using periodic arrays at different Reynolds and frequencies; then, the coefficients of the N-K model were corrected using the simulation results.

\section{Flow equations in a macroscale}

Turbulence can usually be significant at high Reynolds numbers in the porous media. If the Reynolds number is high enough on the microscale and the length scale of the turbulence is considerably lower than that of the pores of the porous media, turbulence models provided for non-porous conditions can be used to solve the flow field in porous media on a microscale [11].

Time-averaged equations including continuity, Navier-Stocks, energy, turbulence kinetic energy, and rate of dissipation equations are as follows:

$$
\begin{aligned}
& \frac{\partial \rho_{f}}{\partial t}+\frac{\partial\left(\rho_{f} \bar{u}_{j}\right)}{\partial x_{j}}=0 \\
& \frac{\partial\left(\rho_{f} \bar{u}\right)}{\partial t}+\frac{\partial\left(\rho_{f} \bar{u}_{j} \bar{u}_{i}\right)}{\partial x_{j}}=-\frac{\partial(\bar{p})}{\partial x_{i}} \\
& \quad+\frac{\partial}{\partial x_{j}}\left(\mu\left(\frac{\partial \bar{u}_{i}}{\partial x_{j}}+\frac{\partial \bar{u}_{j}}{\partial x_{i}}\right)\right)-\rho_{f} \overline{u^{\prime} v^{\prime}}, \\
& \frac{\partial\left(\rho_{f} c_{p f} \bar{T}\right)}{\partial t}+\frac{\partial\left(\rho_{f} c_{p f} \bar{u}_{j} \bar{T}\right)}{\partial x_{j}} \\
& =\frac{\partial}{\partial x_{j}}\left(k_{f} \frac{\partial \bar{T}}{\partial x_{j}}-\rho_{f} c_{p f} \overline{T^{\prime} u_{j}^{\prime}}\right),
\end{aligned}
$$




$$
\begin{aligned}
\frac{\partial\left(\rho_{f} k\right)}{\partial t} & +\frac{\partial\left(\rho_{f} \bar{u}_{j} k\right)}{\partial x_{j}}=\frac{\partial}{\partial x_{j}}\left(\left(\mu+\frac{\mu_{t}}{\sigma_{\epsilon}}\right) \frac{\partial k}{\partial x_{j}}\right) \\
& -\overline{u_{i}^{\prime} u_{j}^{\prime}} \frac{\partial \bar{u}_{j}}{\partial x_{j}}-\epsilon, \\
\frac{\partial\left(\rho_{f} \epsilon\right)}{\partial t}+ & \frac{\partial\left(\rho_{f} \bar{u}_{j} \epsilon\right)}{\partial x_{j}}=\frac{\partial}{\partial x_{j}}\left(\left(\mu+\frac{\mu_{t}}{\sigma_{\epsilon}}\right) \frac{\partial \epsilon}{\partial x_{j}}\right) \\
& +\left(-c_{1} \overline{u_{i}^{\prime} u_{j}^{\prime}} \frac{\partial \bar{u}_{j}}{\partial x_{j}}-c_{2} \epsilon\right) \frac{\epsilon}{k} .
\end{aligned}
$$

The term of Reynolds stress can be modeled using Boussinesq model, as shown in the following:

$$
\rho_{f} \overline{u_{i}^{\prime} u_{j}^{\prime}}=\mu_{t}\left(\frac{\partial \bar{u}_{i}}{\partial x_{j}}+\frac{\partial \bar{u}_{j}}{\partial x_{i}}\right)-\frac{2}{3} \rho_{f} k \delta_{i j}
$$

and:

$$
\rho_{f} c_{p f} \overline{T^{\prime} u_{j}^{\prime}}=c_{p f} \frac{\mu_{t}}{\sigma_{T}} \frac{\partial \bar{T}}{\partial x_{j}} .
$$

Turbulence viscosity can be obtained using Eq. (10):

$$
\mu_{t}=c_{\mu} \frac{k^{2}}{\epsilon} \text {. }
$$

For the solid phase, the energy equation is calculated using Eq. (11):

$$
\frac{\partial\left(\rho_{s} c_{p s} T\right)}{\partial T}=\frac{\partial}{\partial x_{j}}\left(k_{s} \frac{\partial T}{\partial x_{j}}\right) .
$$

The coefficients used in the above equations are calculated as follows:

$$
\begin{aligned}
& c_{\mu}=0.09, \quad c_{1}=1.44, \quad c_{2}=1.92, \\
& \sigma_{k}=1.00, \quad \sigma_{\epsilon}=1.30, \quad \sigma_{T}=0.90 .
\end{aligned}
$$

Eq. (12) uses intrinsic volume average values of turbulence to define the turbulence viscosity.

$$
\mu_{t}=c_{\mu} \frac{\left(\langle k\rangle^{f}\right)^{2}}{\langle\epsilon\rangle^{f}} \text {. }
$$

By integrating $k$ and $\varepsilon$ equations, in a representative elementary volume, the equations would be changed into the one shown in the following [11]:

$$
\begin{aligned}
& \frac{\partial\left(\rho_{f}\langle k\rangle^{f}\right)}{\partial t}+\frac{\partial\left(\rho_{f}\left\langle\bar{u}_{j}\right\rangle^{f}\langle k\rangle^{f}\right)}{\partial x_{j}} \\
& =\frac{\partial}{\partial x_{j}}\left(\left(\mu+\frac{\mu_{t}}{\sigma_{k}}\right) \frac{\partial\langle k\rangle^{f}}{\partial x_{j}}\right)+2 \mu_{t}\left\langle s_{i j}\right\rangle^{f}\left\langle s_{i j}\right\rangle^{f} \\
& \quad-\langle\epsilon\rangle^{f}+2 \mu_{t}\left\langle s_{i j}^{\prime \prime}\right\rangle^{f}\left\langle s_{i j}^{\prime \prime}\right\rangle^{f}+\frac{\mu}{V_{f}} \int_{A_{\text {int }}} \frac{\partial k}{\partial x_{j}} n_{j} d A \\
& -\frac{\partial}{\partial x_{j}}\left\langle\overline{u^{\prime \prime}}{ }_{j} k^{\prime \prime}\right\rangle^{f},
\end{aligned}
$$

$$
\begin{aligned}
& \frac{\partial\left(\rho_{f}\langle\epsilon\rangle^{f}\right)}{\partial t}+\frac{\partial\left(\rho_{f}\left\langle\bar{u}_{j}\right\rangle^{f}\langle\epsilon\rangle^{f}\right)}{\partial x_{j}} \\
& =\frac{\partial}{\partial x_{j}}\left(\left(\mu+\frac{\mu_{t}}{\sigma_{\epsilon}}\right) \frac{\partial\langle\epsilon\rangle^{f}}{\partial x_{j}}\right) \\
& \quad+\left(2 c_{1} \mu_{t}\left\langle s_{i j}\right\rangle^{f}\left\langle s_{i j}\right\rangle^{f}-\langle\epsilon\rangle^{f}\right) \frac{\langle\epsilon\rangle^{f}}{\langle k\rangle^{f}} \\
& \quad+2 c_{1} \mu_{t}\left\langle s_{i j}^{\prime \prime}\right\rangle^{f}\left\langle s_{i j}^{\prime \prime}\right\rangle^{f} \frac{\langle\epsilon\rangle^{f}}{\langle k\rangle^{f}}+\frac{\mu}{V_{f}} \int_{A_{\mathrm{int}}} \frac{\partial \epsilon}{\partial x_{j}} n_{j} d A \\
& \quad-\frac{\partial}{\partial x_{j}}\left\langle\bar{u}^{\prime \prime}{ }_{j} \epsilon^{\prime \prime}\right\rangle^{f}, \\
& s_{i j}=\frac{1}{2}\left(\frac{\partial \bar{u}_{i}}{\partial x_{j}}+\frac{\partial \bar{u}_{j}}{\partial x_{i}}\right)
\end{aligned}
$$

In the above equations, the third- and higher-order terms are omitted. Due to the presence of the porous media, two extra terms were created including the production term $2 \mu_{t}\left\langle s_{i j}^{\prime \prime}\right\rangle^{f}\left\langle s_{i j}^{\prime \prime}\right\rangle^{f}$ and dissipation rate $\frac{\mu}{V_{f}} \int_{A_{\text {int }}} \frac{\partial k}{\partial x_{j}} n_{j} d A$. The $2 \mu_{t}\left\langle s_{i j}^{\prime \prime}\right\rangle^{f}\left\langle s_{i j}^{\prime \prime}\right\rangle^{f}$ term represents the production of the turbulence kinetic energy for a laminar uniform flow without shear stress, and the $\int_{A_{\text {int }}} \frac{\partial k}{\partial x_{j}} n_{j} d A$ part of the dissipation term is negative as a result of the no-slippage condition. The sum of these two terms shows the net production in the presence of porosity. Therefore, these two terms can be modeled as shown in the following:

$$
\epsilon_{\infty}=2 \mu_{t}\left\langle s_{i j}^{\prime \prime}\right\rangle^{f}\left\langle s_{i j}^{\prime \prime}\right\rangle^{f}+\frac{\mu}{V_{f}} \int_{A_{\mathrm{int}}} \frac{\partial k}{\partial x_{j}} n_{j} d A .
$$

These terms are modeled in the equation of turbulence energy dissipation rate, as shown in the following:

$$
c_{2} \frac{\epsilon_{\infty}^{2}}{k_{\infty}}=2 c_{1} \mu_{t}\left\langle s_{i j}^{\prime \prime}\right\rangle^{f}\left\langle s_{i j}^{\prime \prime}\right\rangle^{f} \frac{\langle\epsilon\rangle^{f}}{\langle k\rangle^{f}}+\frac{\mu}{V_{f}} \int_{A_{\text {int }}} \frac{\partial \epsilon}{\partial x_{j}} n_{j} d A .
$$

Experimental determination of unknown constants $k$ and $\varepsilon$ needs complex and accurate measurements on the pore scale; accordingly, these constants are obtained using numerical experiments. For a macroscopic flow without shear stress, $k$ and $\varepsilon$ equations are simplified, as shown below:

$$
\begin{aligned}
& \langle\bar{u}\rangle^{f} \frac{\partial\left(\langle k\rangle^{f}\right)}{\partial x}=-\langle\epsilon\rangle^{f}+\epsilon_{\infty}, \\
& \langle\bar{u}\rangle^{f} \frac{\partial\left(\langle\epsilon\rangle^{f}\right)}{\partial x}=-c_{2} \frac{\left(\langle\epsilon\rangle^{f}\right)^{2}}{\langle k\rangle^{f}}+c_{2} \frac{\epsilon_{\infty}^{2}}{k_{\infty}} .
\end{aligned}
$$

By neglecting the velocity gradient in the $X$ direction, the equation is simplified as shown in the following: 


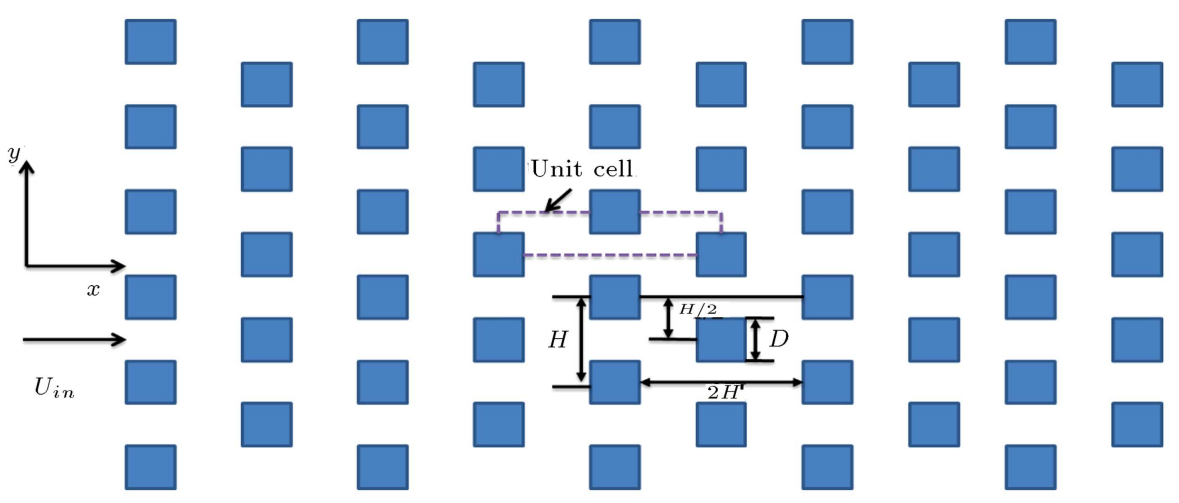

Figure 2. Periodic arrays for modeling an oscillating flow in porous media on a microscale.

$$
\langle k\rangle^{f}=k_{\infty}, \quad\langle\epsilon\rangle^{f}=\epsilon_{\infty} .
$$

Therefore, by simulating the flow on the microscale, constants in the equations above can be obtained. Kuwahara et al. (1998) [26] and Kundu et al. (2016) [27] conducted several numerical experiments using periodic arrays in square, circular, cubic, and spherical shapes in two-dimensional and three-dimensional forms; in addition, similar results from calculating the permeability in the laminar regime were achieved for each array. Two arrangements of arrays are considered for numerical investigation in the porous media. These two arrangements are in line and staggered arrangement. In most prior studies, staggered arrangements were selected due to their capability in capturing nonhomogenous and random structure of porous media physics in real-world conditions. In this regard, twodimensional staggered square arrays were used to model the flow on a microscale. Figure 2 shows these arrays used for simulating turbulent oscillating flow on a microscale. The porosity of these arrays can be calculated using the following equation:

$$
\varepsilon=1-\left(\frac{D}{H}\right)^{2} .
$$

Prior studies showed that both inlet and outlet effects of the flow were eliminated by choosing a proper inlet and outlet length. Kim suggested $2 H$ and $7 H$ for the inlet and outlet lengths, respectively, to eliminate the boundary condition at the outlet [28].

\section{Numerical method}

Researchers have considered the periodic boundary conditions to study the details of flow in a unit cell. However, in an oscillating flow, a unit cell is insufficient to study the input effects and phase difference of the flow using periodic boundary conditions in the direction of the flow. Therefore, to study an oscillating
Figure 3. Periodic array chosen to simulate oscillating turbulent flow in porous media.

flow, seven duplicated cells are used [29]. The symmetry boundary condition is used in the upper and lower parts of each cell to minimize the computational cost, which is acceptable due to the symmetry of the flow to the $x$-axis. Figure 3 shows the periodic array used for simulating the turbulent oscillating flow in a porous environment. Boundary conditions are considered symmetrical in the upper and lowers parts of the periodic array (Eq. (22)). In addition, fully developed boundary conditions are applied (Eq. (23)) to the tail of this array. Moreover, the velocity at the inlet of the array is considered sinusoidal, as defined in Eq. (24). The well-established SIMPLE method is used to deal with pressure and velocity coupling. Collocated arrangement of the grid is also employed to discretize the aforementioned equations. Rhie-Chow interpolation technique is utilized to handle the checker board in the pressure field [30,31].

$$
\begin{aligned}
& \frac{\partial u}{\partial y}=0, \quad \frac{\partial v}{\partial y}=0, \\
& \frac{\partial u}{\partial x}=0, \quad \frac{\partial v}{\partial x}=0 \\
& u_{i n}=U_{i n} \sin (2 \pi f \times t) .
\end{aligned}
$$

Based on the inlet velocity, Reynolds is considered at its maximum [2]:

$$
\operatorname{Re}_{\max }=\frac{\rho_{f} U_{i n} d_{p}}{\mu_{f}} .
$$

Figure 4 shows the cell units for $0.305,0.49,0.61$, $0.69,0.75$, and 0.826 porosities. The standard $k-\varepsilon$ model was used for simulating the flow. The equations were discretized by the finite volume technique. The 


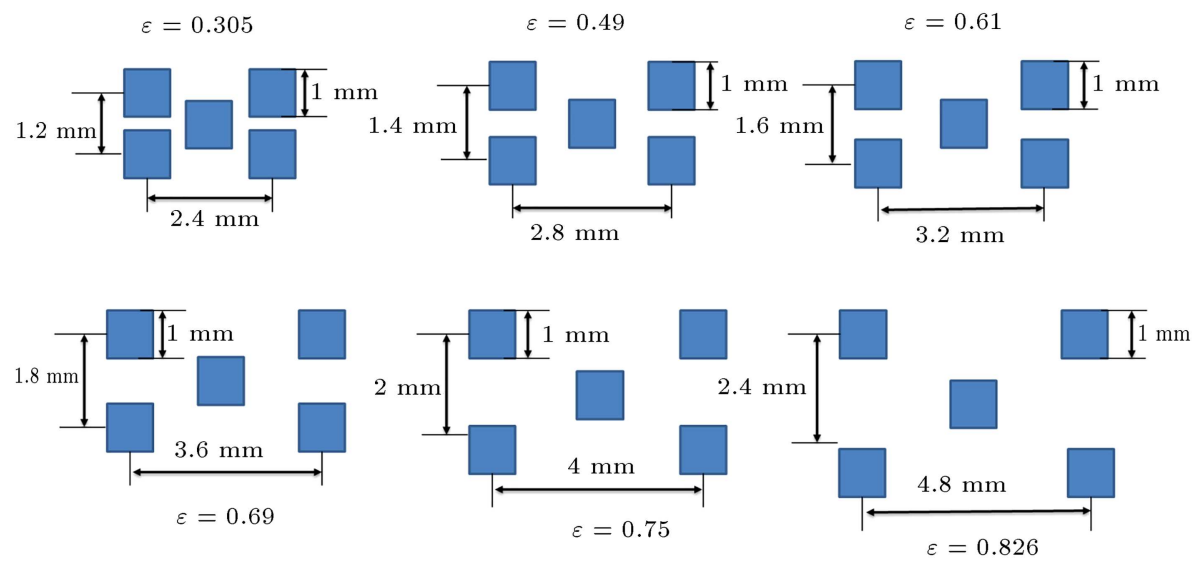

Figure 4. Periodic arrays for $0.305,0.49,0.61,0.69,0.75$, and 0.826 porosities.

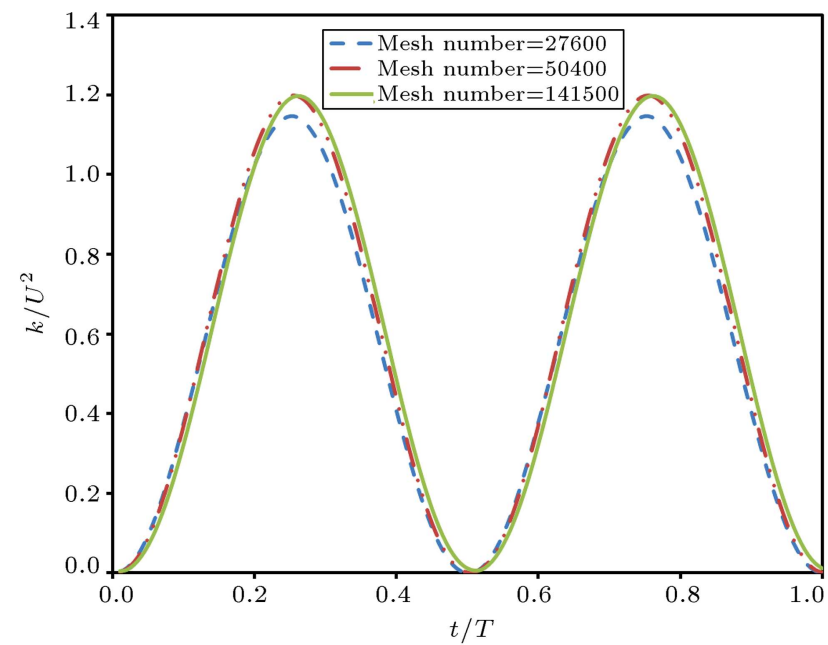

Figure 5. Dimensionless kinetic energy in periodic arrays versus time for three solution grids in a cycle.

$\operatorname{Re}_{p, \max }=3200, f=1 \mathrm{~Hz}, \varepsilon=0.69$.

SIMPLE method was adopted to handle the pressure and velocity coupling. The algorithm starts with two momentum equations. The pressure was corrected by solving the pressure correction equation. Then, $k-\varepsilon$ equations employed to calculate the turbulent viscosity were solved. The TDMA algorithm was used to solve these algebraic sets of equations [32].

To study the independency of the solution from the grid on a macroscale, three grids each with 27600 , 50400 , and 141500 cells were employed to solve the flow at $\operatorname{Re}_{p, \max }=3200, f=1 \mathrm{~Hz}$ frequency, and porosity of $\varepsilon=0.69$. Figures 5 and 6 depict the average turbulence kinetic energy and turbulence energy dissipation rate in a periodic array for these three solution grids. As observed earlier, two finer grids produce similar results. Therefore, the 50400 grid is suitable for solving the flow. This method was used for other Reynolds and frequencies to check the grid independency to obtain an optimum grid number.

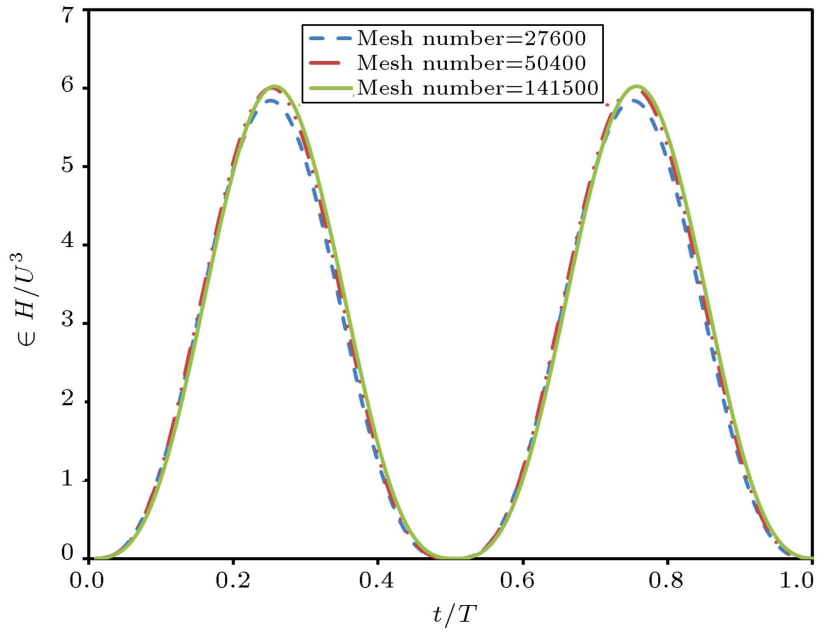

Figure 6. Dimensionless dissipation rate of kinetic energy in periodic arrays over time for three solution grids in a cycle. $\operatorname{Re}_{p, \max }=3200, f=1 \mathrm{~Hz}, \varepsilon=0.69$.

To check the independency of the solution on the time step, three-time steps were taken into account. Figures 7 and 8 demonstrate the averaged turbulence kinetic energy and turbulence energy dissipation rate in a periodic array for an oscillating flow with $\operatorname{Re}_{p, \max }=$ $3200, f=1 \mathrm{~Hz}$ frequency, and $\varepsilon=0.69$ porosity. As shown, choosing a time step of $0.001 \mathrm{~s}$ is enough to solve the flow. The time step is similarly chosen at other frequencies. The results were compared with those obtained by $\mathrm{Tu}$ and Ramaprian in terms of validation [33]. They measured the velocity profile of the oscillating flow in the fully developed region of a pipe experimentally. The test section containing velocity measurements was a Plexiglas tube, $0.3 \mathrm{~m}$ in length and $50 \mathrm{~mm}$ in the internal diameter. A rotating profiled sleeve driven by a regulated geared D.C. motor controlled the exit area for the water. The sleeve profile was designed to provide two complete cycles of sinusoidal oscillation in discharge in one revolution. As 


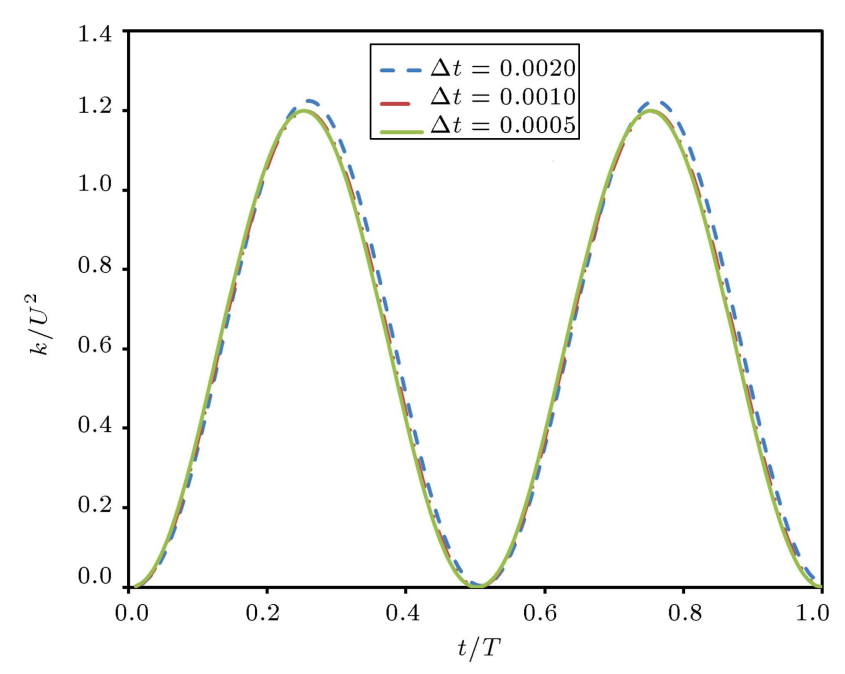

Figure 7. Dimensionless kinetic energy in periodic arrays versus time for three time steps in a cycle.

$\operatorname{Re}_{p, \max }=3200, f=1 \mathrm{~Hz}, \varepsilon=0.69$.

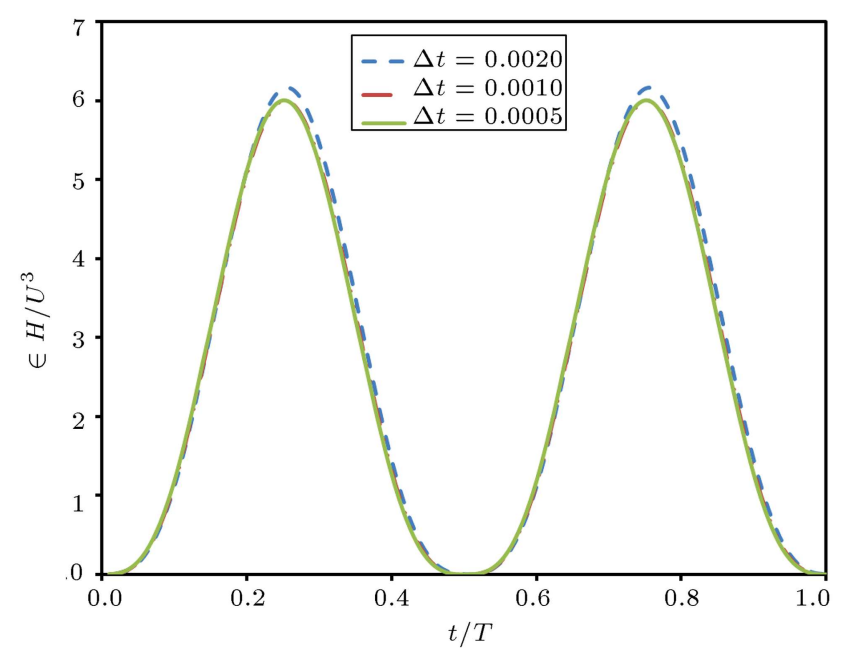

Figure 8. Dimensionless dissipation rate of kinetic energy in periodic arrays versus time for three time steps in a cycle. $\operatorname{Re}_{p, \max }=3200, f=1 \mathrm{~Hz}, \varepsilon=0.69$.

shown in Figure 9, there is good agreement between the results of the present code and experiments, and this code can be practically used for oscillating flow simulation.

Figures 10-14 present the velocity, pressure, turbulence kinetic energy, dissipation rate, and turbulent viscosity contours at $T / 4, T / 2,3 T / 4$, and $T$ times $(T$ is the time period of a cycle). Except in the first and last cells, the velocity contour was repeated in other cells at each time step. In order to calculate the values of time-varying turbulence such as turbulent kinetic energy and its dissipation, a central cell should be chosen and its average amount in a cycle was obtained by surface averaging. Contours are shown enlarged in Figures 15-18. A vortex formed at the corner of the cells due to abrupt changes in the flow

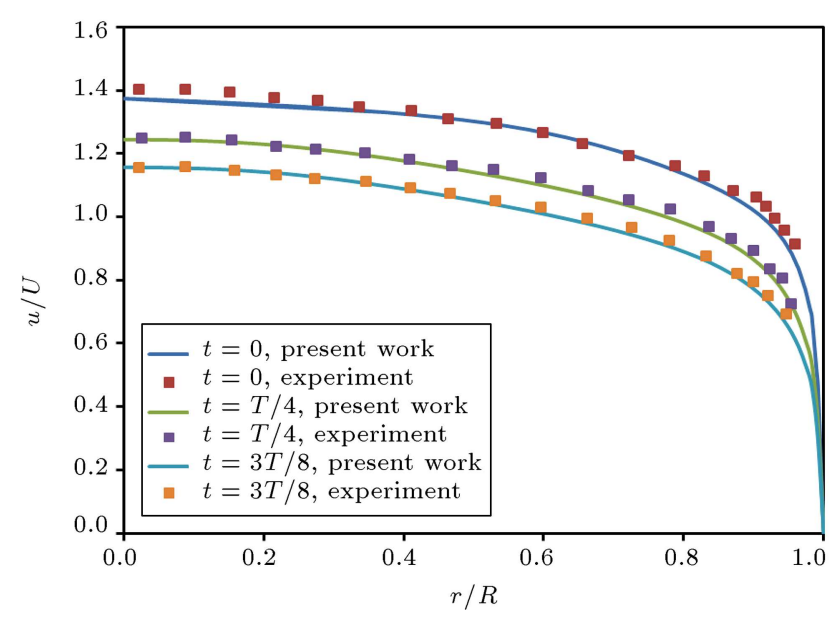

Figure 9. Code validation: Comparison of the velocity profile in a fully developed turbulent oscillating flow in one period with that obtained by Tu and Ramaprian [33].

direction. The vortexes formed due to flow separation and adverse pressure gradient in an abrupt expansion. In the first half cycle, the vortex was symmetrically placed by the second half cycle. In the dead zone of the piston, prolate vortexes formed in the entire flow field in the first and second halves of the cycle due to the piston returning and drawing part of the flow with it. The other part of the flow moved in the opposite direction to the piston movement because of inertia, thus forming two opposing vortexes. In areas far from the walls and within the symmetrical boundaries, the velocity was at its maximum which could form boundary layers by the walls. Figure 11 shows the fluid pressure decline proportional to the length of the periodic array. Maximum pressure drop was observed, while the fluid velocity in the periodic array placed the Reynolds in the Forchheimer regime. At lower Reynolds and Darcy regime, pressure drop was highly affected by fluid viscosity and thus, pressure drop was lower than that in the Forchheimer regime. While fluid pressure declined by the square of fluid velocity in the Forchheimer regime, the relation between the two is linear in the Darcy regime.

Figures 19 and 20 show the turbulence kinetic energy and its dissipation rate for $\operatorname{Re}_{p, \max }=3200$ and $\varepsilon=0.69$. As observed, frequency had no effect on the turbulence kinetic energy and its dissipation rate. Figure 21 depicts the constant coefficients of the turbulence kinetic energy and its dissipation rate for $\operatorname{Re}_{p}=800$ and $\operatorname{Re}_{p}=1600$. Reynolds number can easily affect the turbulence energy and its dissipation rate. Upon increasing the Reynolds number, the correlation coefficient of the turbulence kinetic energy increased in the N-K model, while that of the dissipation rate of turbulence energy decreased.

In Figures 22 and 23, the correlation coefficients are plotted versus Reynolds. Upon increasing the 


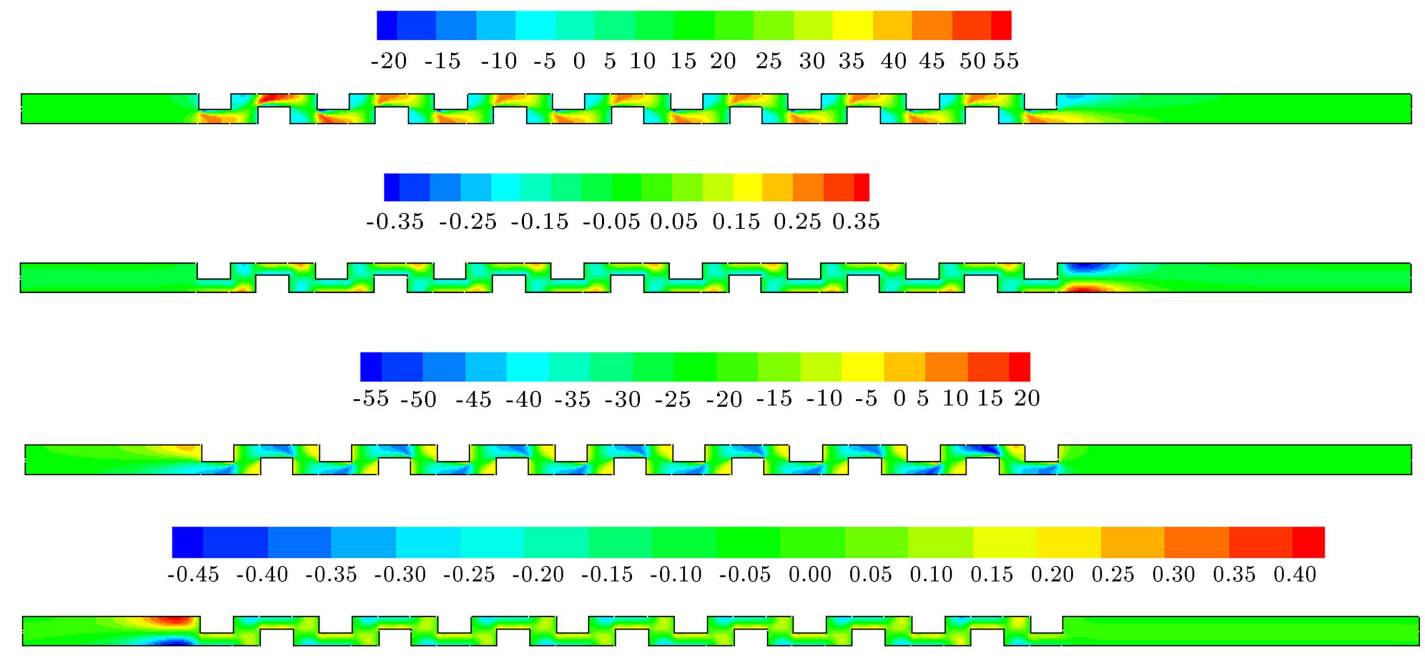

Figure 10. Velocity contours at three times, $T / 4, T / 2,3 T / 4$, and $T$. $\operatorname{Re}_{p, \max }=3200, f=1 \mathrm{~Hz}$, and $\varepsilon=0.69$ shown in the figure.

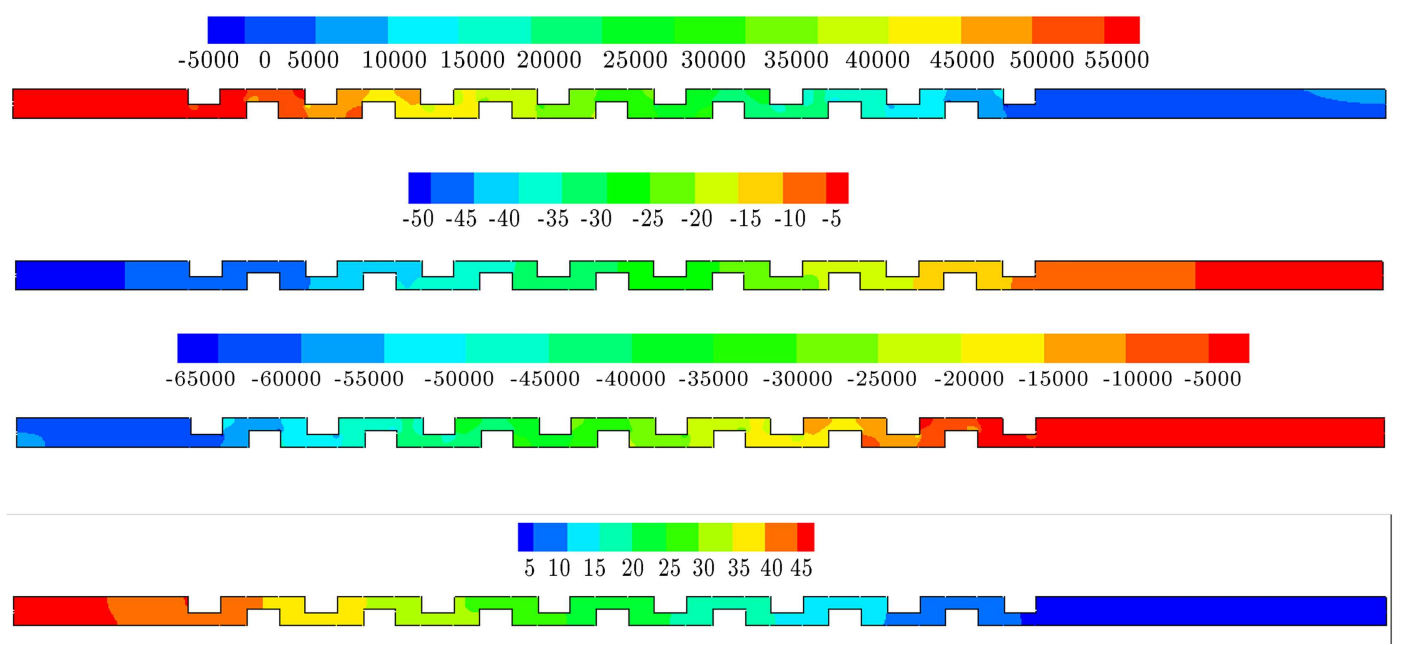

Fig ure 11. Pressure $(\mathrm{Pa})$ contours at three times, $T / 4, T / 2,3 T / 4$, and $T$. $\operatorname{Re}_{p, \max }=3200, f=1 \mathrm{~Hz}, \varepsilon=0.69$.

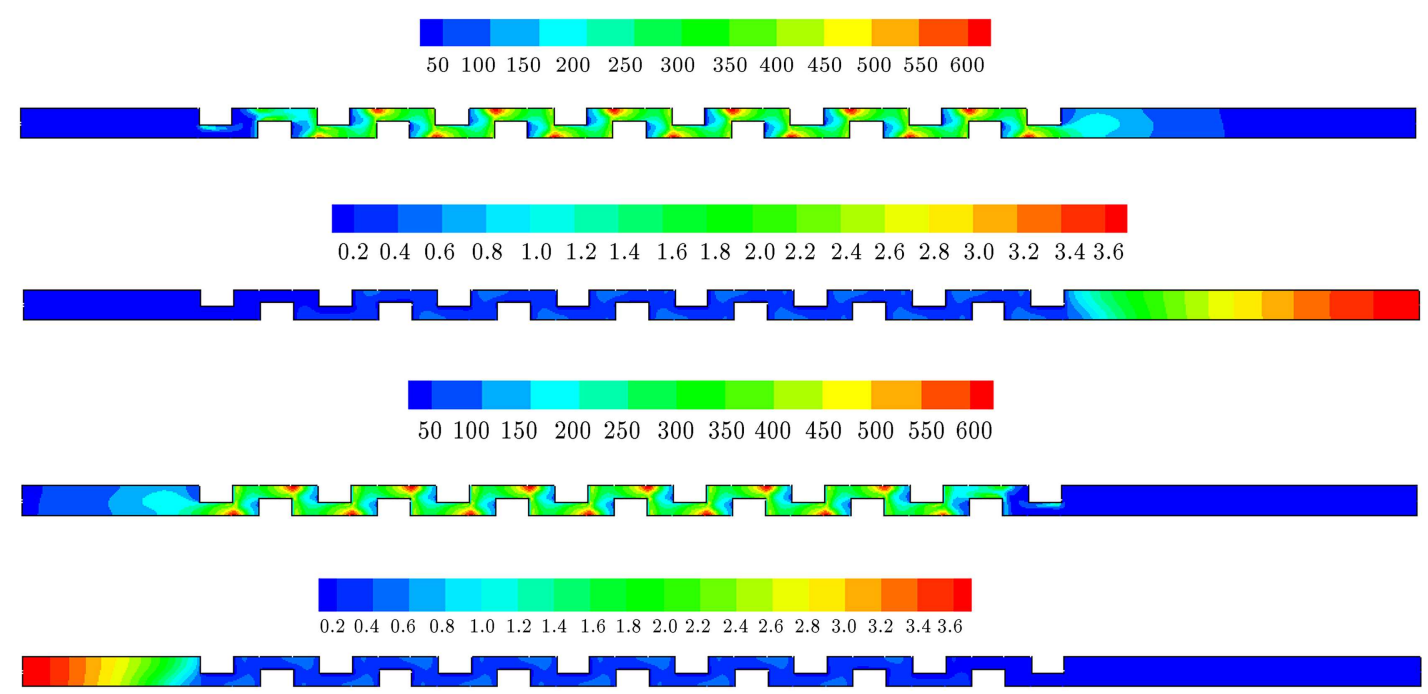

Figure 12. Turbulence kinetic energy $\left(\mathrm{m}^{2} / \mathrm{s}^{2}\right)$ contours at three times, $T / 4, T / 2,3 T / 4$, and $T$. $\operatorname{Re}_{p, \max }=3200, f=1 \mathrm{~Hz}$, $\varepsilon=0.69$. 


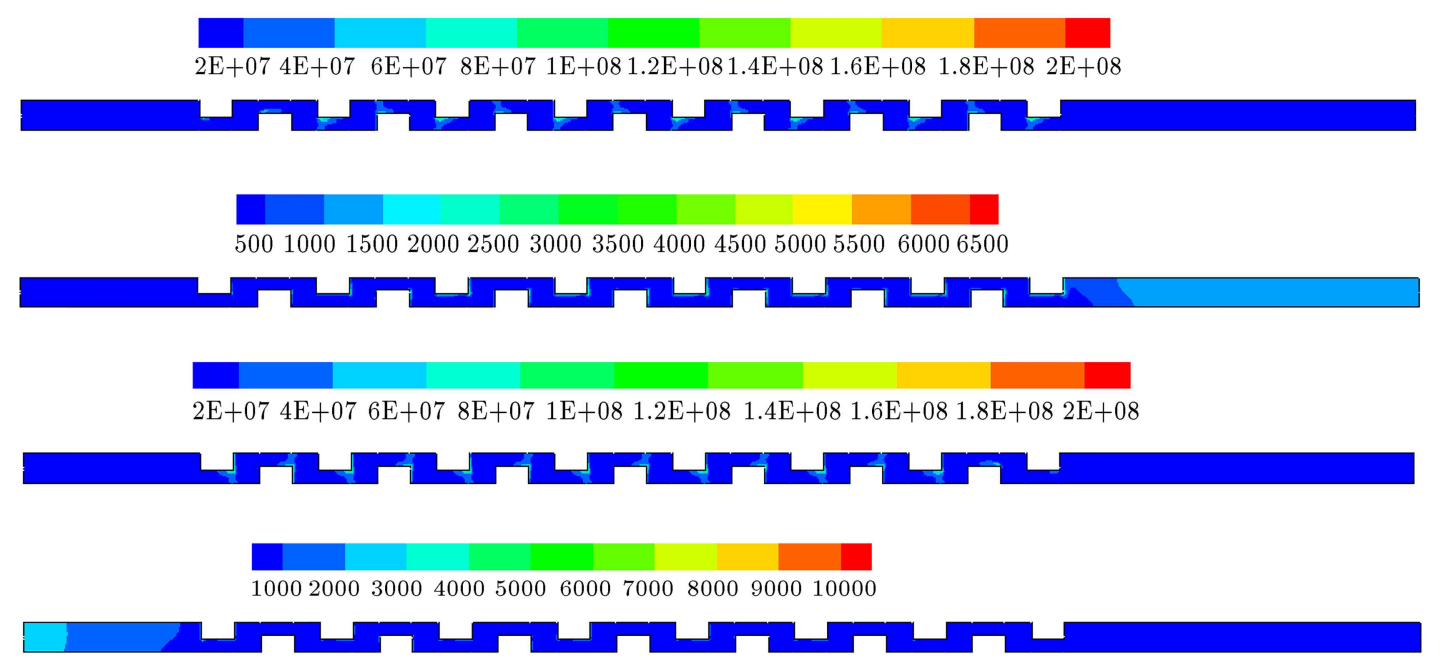

Figure 13. Dissipation rate of turbulence kinetic energy $\left(\mathrm{m}^{2} / \mathrm{s}^{3}\right)$ contours at three times, $T / 4, T / 2,3 T / 4$, and $T$. $\operatorname{Re}_{p, \max }=3200, f=1 \mathrm{~Hz}, \varepsilon=0.69$.

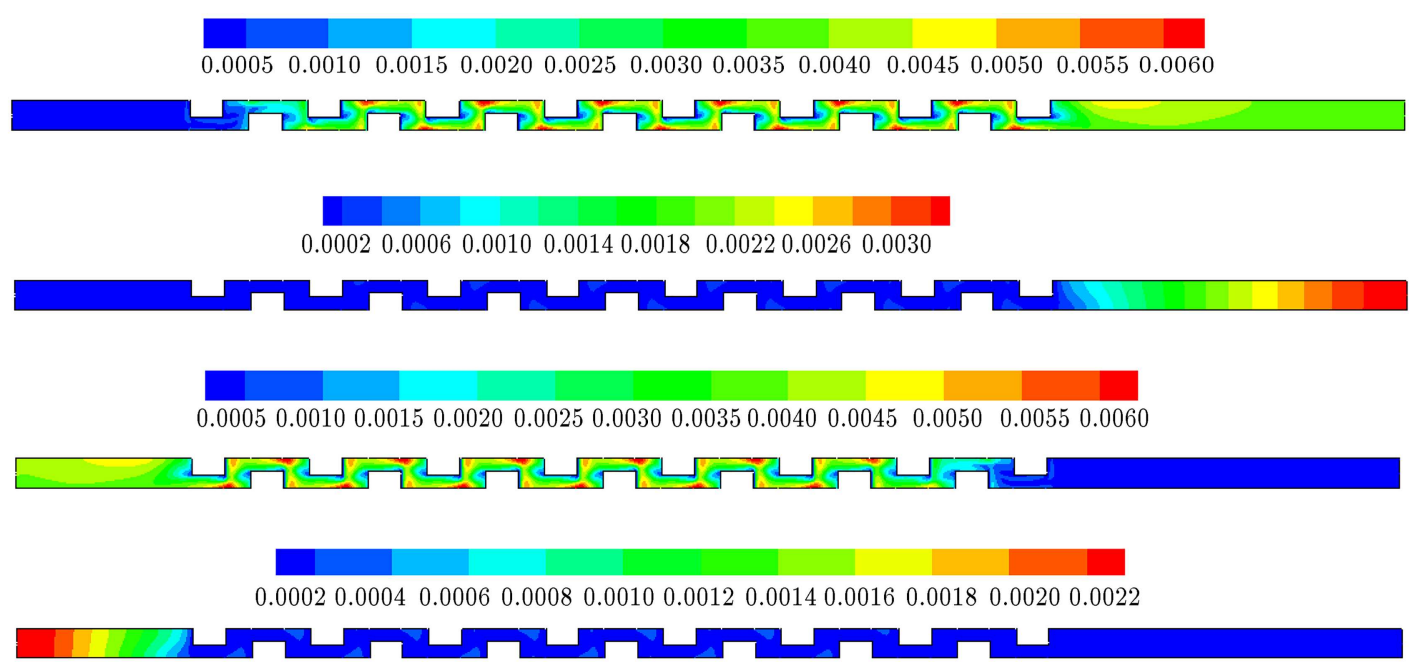

Figure 14. Turbulent viscosity (kg/m.s) contours at three times, $T / 4, T / 2,3 T / 4$, and $T \cdot \operatorname{Re}_{p, \max }=3200, f=1 \mathrm{~Hz}$, $\varepsilon=0.69$.

Reynolds numbers, the above coefficients of the turbulence kinetic energy and dissipation rate increased and decreased, respectively. The coefficients of both equations behaved asymptotically and while increasing, they became independent of the Reynolds number. An equation was suggested for each of the coefficients. For turbulence kinetic energy and its dissipation rate, the mentioned coefficient equation is given, as shown in Eqs. (26) and (27).

$$
\begin{aligned}
& \text { coefficient }=3.94-\frac{16.34}{\operatorname{Re}_{p, \max }^{0.4}}, \\
& \text { coefficient }=48.98+\frac{1.241 \times 10^{6}}{\operatorname{Re}_{p, \max }^{1.7}} .
\end{aligned}
$$

To validate the results of the corrected model, the channel flow of the porous media was modeled using volumetrically averaged equations and then, the obtained results were compared with those of the microscale and N-K models. Figures 24 and 25 represent the turbulence kinetic energy and its dissipation rate in one cycle. The corrected model yielded better results than the N-K model. The coefficients of the N-K model were provided for higher Reynolds numbers and in such conditions, those coefficients were independent of the Reynolds number. However, in many applications, the Reynolds number of the flow was lower than what Kuwahara and Nakayama considered for their proposed model. Therefore, these coefficients should be corrected before use in other Reynolds numbers. Figures 24 and 25 suggest that the corrected model has a better correlation with the microscale model.

\section{Conclusion}

One of the most applied turbulence models in the 


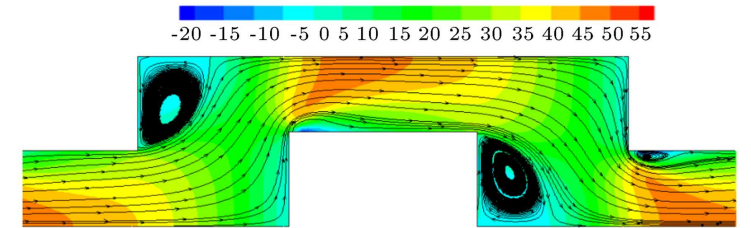

$-0.35-0.30-0.25-0.20-0.15-0.10-0.050 .000 .050 .100 .150 .200 .250 .300 .350 .40$

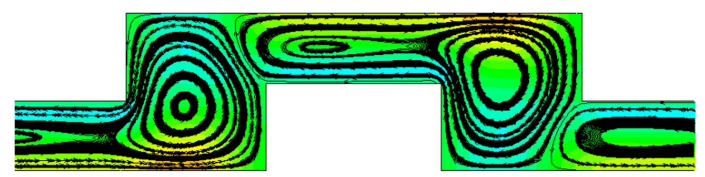

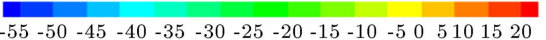

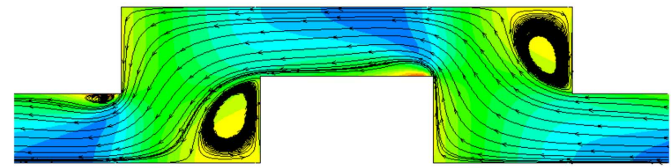

$-0.45-0.40-0.35-0.30-0.25-0.20-0.15-0.10-0.050 .000 .050 .100 .150 .200 .250 .300 .350 .40$

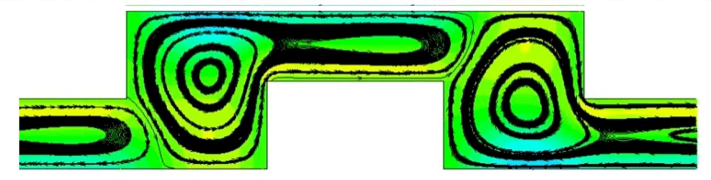

Figure 15. Velocity $(\mathrm{m} / \mathrm{s})$ contours at three times, $T / 4$, $T / 2,3 T / 4$, and $T$. $\operatorname{Re}_{p, \max }=3200, f=1 \mathrm{~Hz}$, and $\varepsilon=0.69$ in one periodic cell.
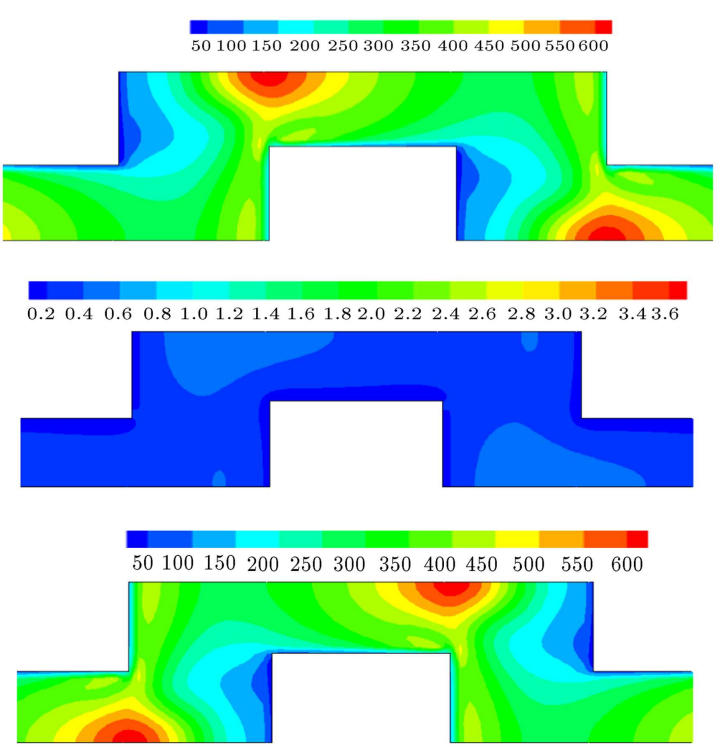

$\begin{array}{llllllllllllllllllll}0.2 & 0.4 & 0.6 & 0.8 & 1.0 & 1.2 & 1.4 & 1.6 & 1.8 & 2.0 & 2.2 & 2.4 & 2.6 & 2.8 & 3.0 & 3.2 & 3.4 & 3.6\end{array}$

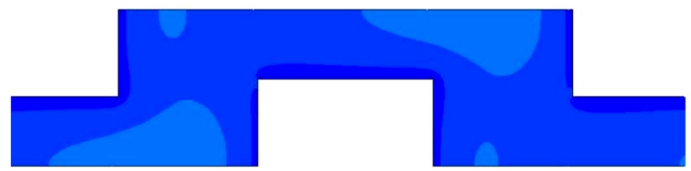

Figure 16. Turbulence kinetic energy $\left(\mathrm{m}^{2} / \mathrm{s}^{2}\right)$ contours at three times, $T / 4, T / 2,3 T / 4$, and $T$. $\operatorname{Re}_{p, \max }=3200$, $f=1 \mathrm{~Hz}$, and $\varepsilon=0.69$ in one periodic cell.

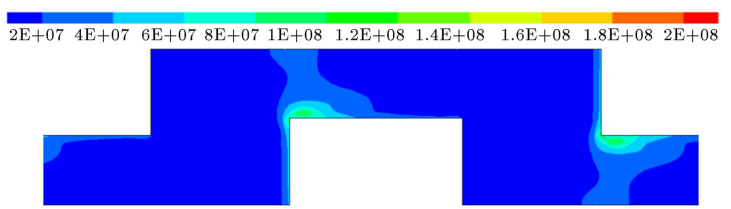

$500100015002000250030003500 \quad 400045005000550060006500$
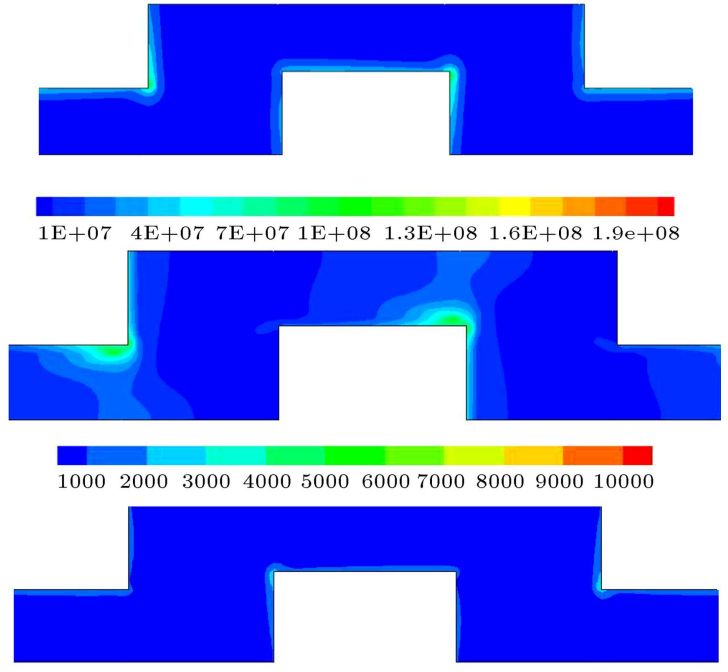

Figure 17. Dissipation rate of turbulent kinetic energy $\left(\mathrm{m}^{2} / \mathrm{s}^{3}\right)$ contours at three times, $T / 4, T / 2,3 T / 4$, and $T$. $\operatorname{Re}_{p, \max }=3200, f=1 \mathrm{~Hz}$, and $\varepsilon=0.69$ in one periodic cell.
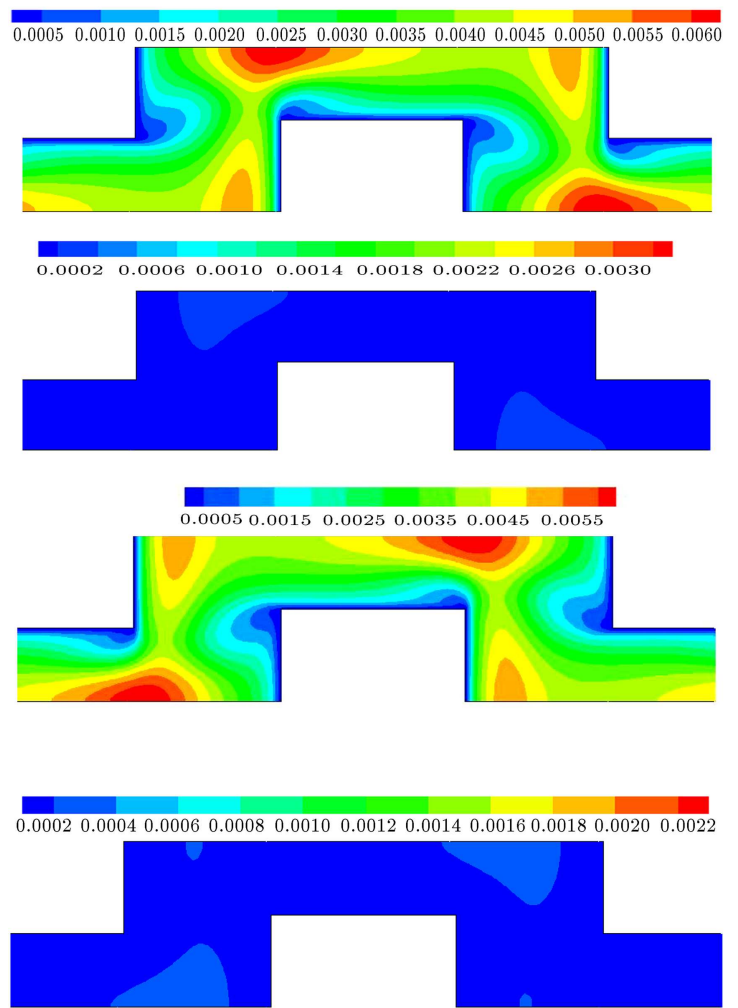

Figure 18. Turbulent viscosity contours $(\mathrm{kg} / \mathrm{m} . \mathrm{s})$ at three times, $T / 4, T / 2,3 T / 4$, and $T$. $\operatorname{Re}_{p, \max }=3200$, $f=1 \mathrm{~Hz}$, and $\varepsilon=0.69$ in one periodic cell many contours. 


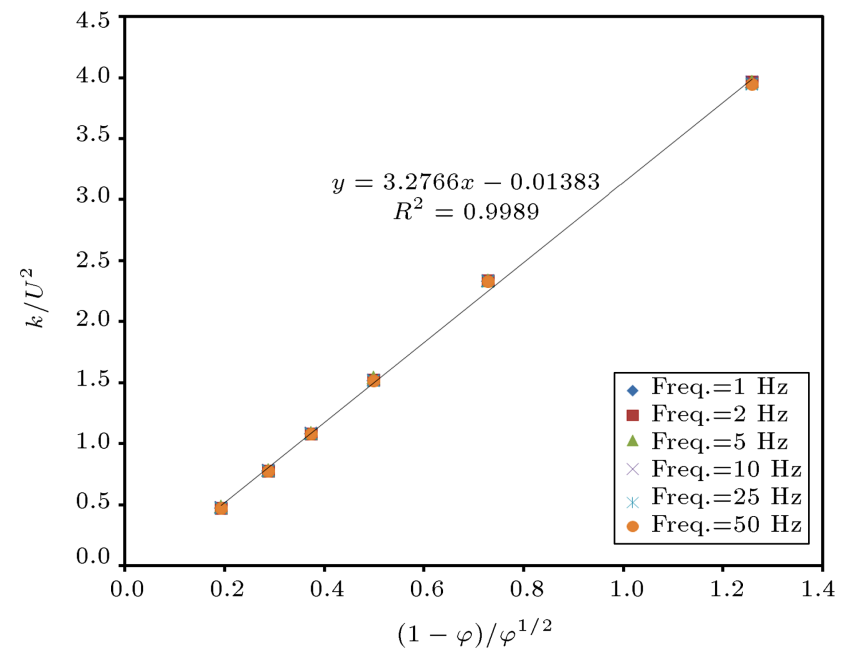

Figure 19. Dimensionless turbulence kinetic energy versus porosity at $\operatorname{Re}_{p}=3200$ at different frequencies.

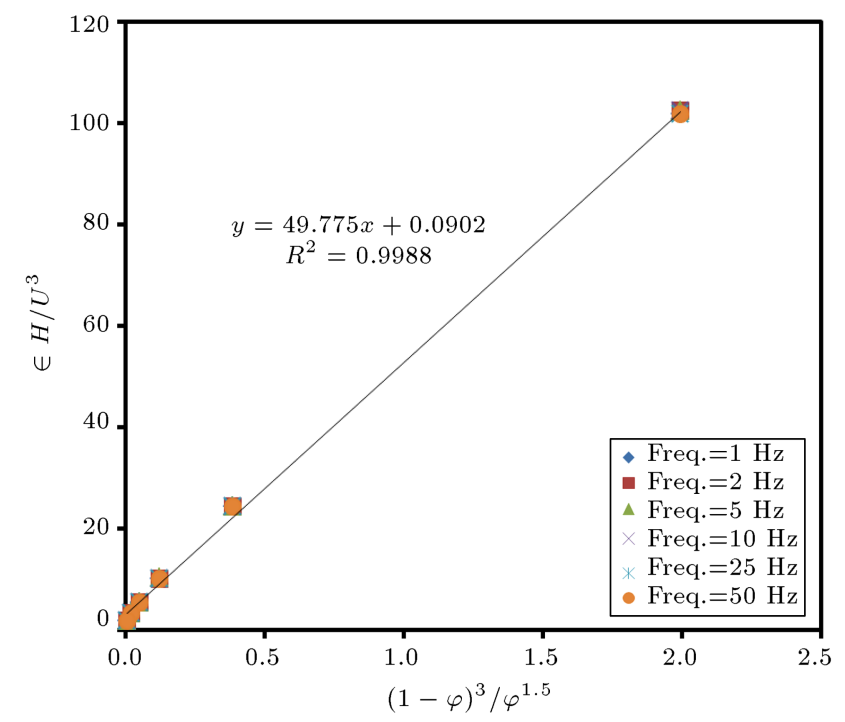

Figure 20. Dimensionless dissipation rate of turbulence kinetic energy diagram over porosity at $\operatorname{Re}_{p}=3200$ at different frequencies.

porous media was proposed by Nakayama and Kuwahara. N-K model was based on the unidirectional and high Reynolds number of the flow in porous media, yet not applicable to the turbulent oscillating flow in the porous media; to this end, a modification to this model was made in this paper. Hence, the turbulent oscillating flow in the porous media on micro and macroscales was numerically investigated. Turbulent flow on the microscale was simulated using standard $k-\varepsilon$ model. The equations were discretized through the control volume approach and the well-established SIMPLE algorithm was used to deal with the pressure and velocity coupling. The N-K model constants were modified to solve the turbulent oscillating flow in the porous media using microscale results. The effect of different parameters on oscillating flow such
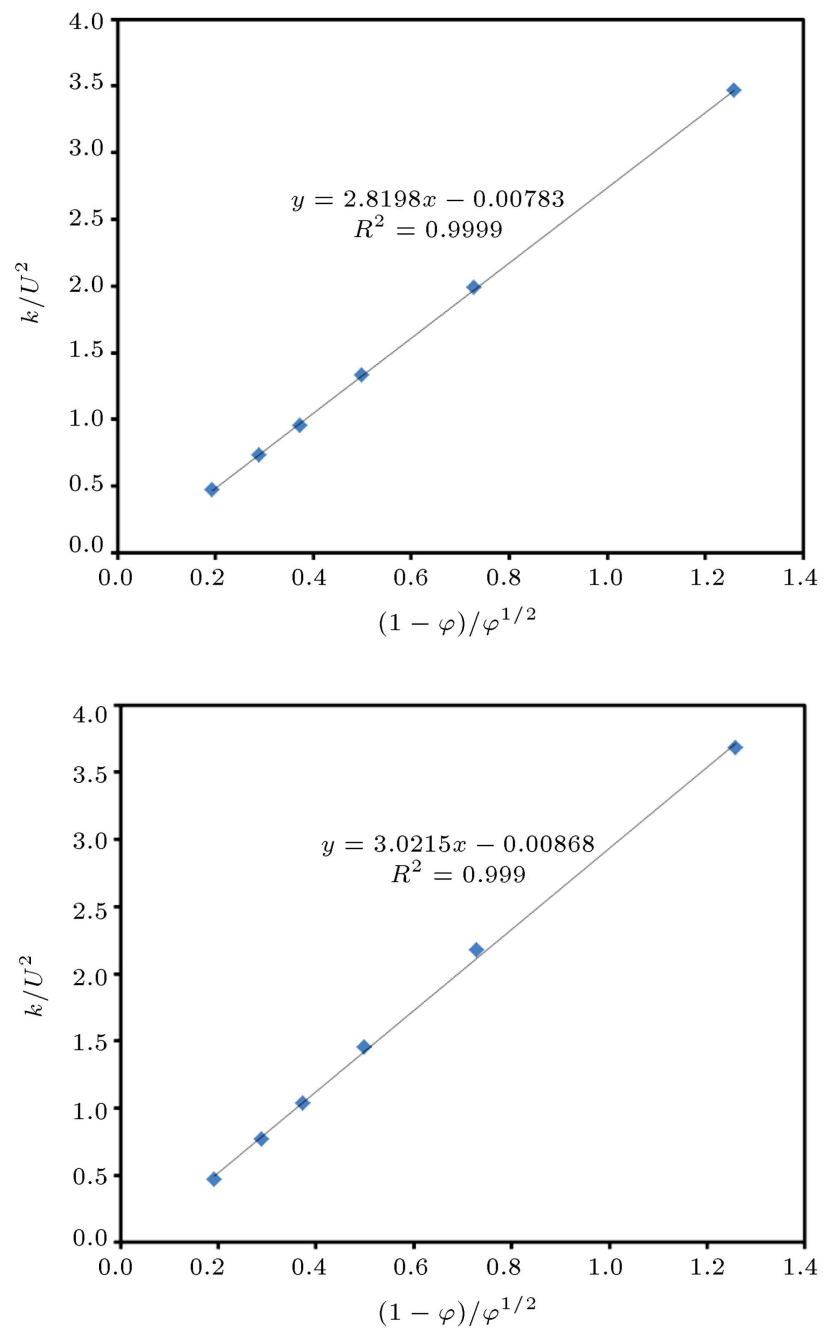

Figure 21. Dimensionless turbulence kinetic energy versus porosity at (a) $\operatorname{Re}_{p}=800$ and $f=1 \mathrm{~Hz}$ and (b) $\operatorname{Re}_{p}=1600$ and $f=1 \mathrm{~Hz}$.

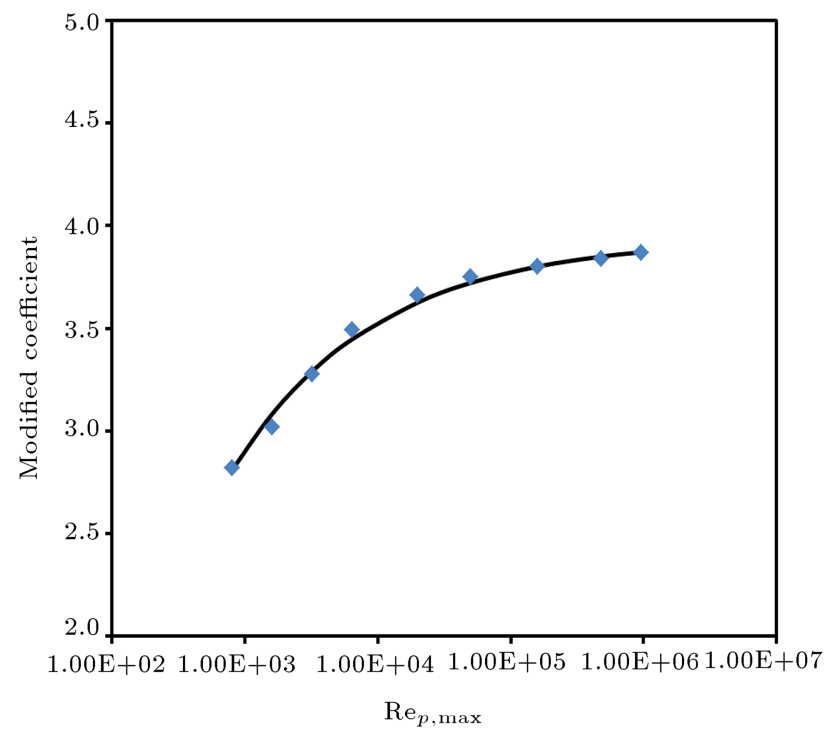

Figure 22. Turbulence kinetic energy correction coefficient versus $\operatorname{Re}_{p, \text { max }}$. 


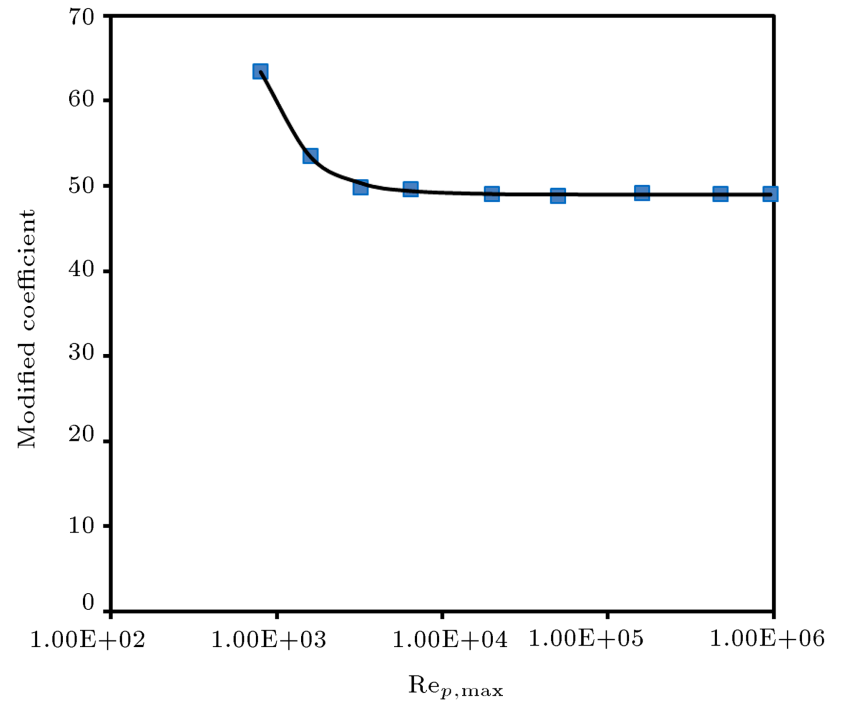

Figure 23. Dissipation rate of turbulent kinetic energy correction coefficient versus $\mathrm{Re}_{p, \max }$.

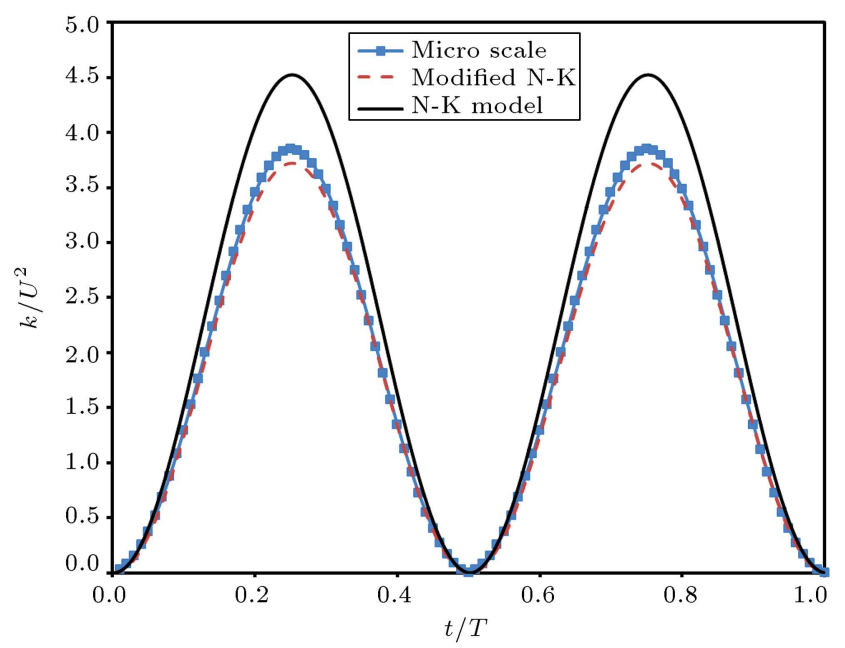

Figure 24. Dimensionless turbulence kinetic energy in one cycle versus time at $\operatorname{Re}_{p}=800, f=1 \mathrm{~Hz}$, and $\varphi=0.305$.

as frequency and Re was also studied. It was shown that frequency had no effect on the constants of the N$\mathrm{K}$ model. However, these constants in the turbulence kinetic energy and its dissipation rate were modified according to the Reynolds number. Two equations for these constants were proposed. The modified model for simulating the turbulent oscillating flow in porous media was verified by simulating the flow with volume averaged equations. The results of the modified model were in line with the N-K original model.

\section{Nomenclature}

$c_{p} \quad$ Specific heat capacity

$d_{p} \quad$ Pore scale diameter

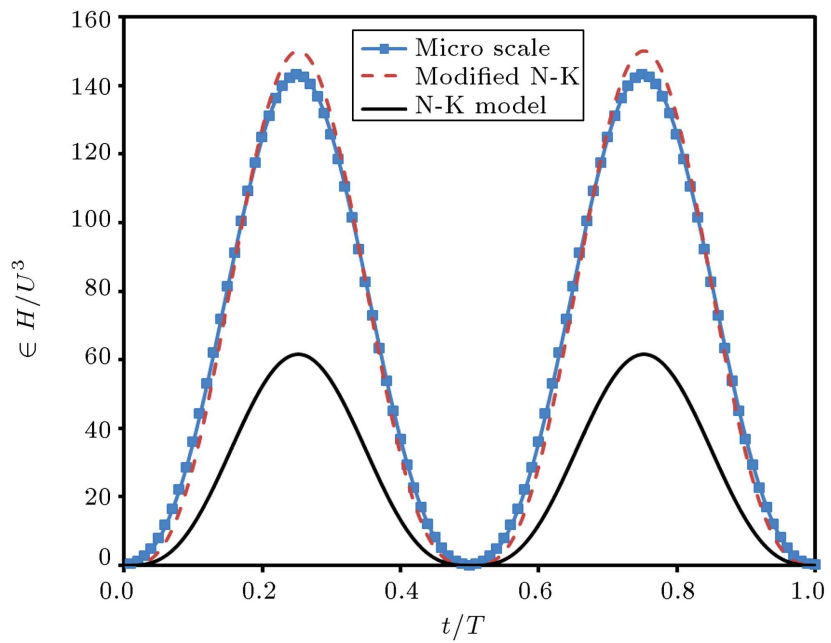

Figure 25. Dimensionless dissipation rate of turbulence kinetic energy in one cycle versus time at $\operatorname{Re}_{p}=800$, $f=1 \mathrm{~Hz}$, and $\varphi=0.305$.

$\begin{array}{ll}D & \text { Unit cell length } \\ f & \text { Frequency } \\ k & \text { Turbulent kinetic energy } \\ H & \text { Unit cell distance } \\ p & \text { Pressure } \\ \operatorname{Re} & \text { Reynolds number } \\ T & \text { Temperature } \\ u & x \text {-velocity } \\ v & y \text {-velocity }\end{array}$

\section{Greek letters}

$\mu \quad$ Dynamic viscosity

$\epsilon \quad$ Turbulent energy dissipation rate

$\varphi \quad$ Porosity

$\rho \quad$ Density

\section{Subscripts}

$f \quad$ Fluid

$\infty \quad$ Variable in fully developed region

$s \quad$ Solid

\section{References}

1. Taherzadeh, M. and Saidi, M.S. "Natural circulation in vertical porous annular enclosure with heat generation", Sci. Iran., 22(1), pp. 208-219 (2015).

2. Kardgar, A. and Jafarian, A. "Numerical investigation of oscillating conjugate heat transfer in pulse tubes", Appl. Therm. Eng., 105, pp. 557-565 (2016).

3. Jolls, K.R. and Hanratty, T.J. "Transition to turbulence for flow through a dumped bed of spheres", Chemical Engineering Science, 21(12), pp. 1185-1190 (1966). 
4. Dybbs, A. and Edwards, R.V. "A new look at porous media fluid mechanics - Darcy to turbulent", in Fundamentals of Transport Phenomena in Porous Media, Dordrecht: Springer Netherlands, pp. 199-256 (1984).

5. Horton, N.A. and Pokrajac, D. "Onset of turbulence in a regular porous medium: An experimental study", Phys. Fluids, 21(4), pp. 045104-045113 (2009).

6. Teruel, F.E. and Uddin, R. "A new turbulence model for porous media flows. Part I: Constitutive equations and model closure", Int. J. Heat Mass Transf., 52(1920), pp. 4264-4272 (Sep. 2009).

7. Masuoka, T. and Takatsu, Y. "Turbulence model for flow through porous media", Int. J. Heat Mass Transf., 39(13), pp. 2803-2809 (1996).

8. Nield, D.A. "Alternative models of turbulence in a porous medium, and related matters", J. Fluids Eng. Trans., ASME, 123(4), pp. 928-934 (2001).

9. Alvarez, G., Bournet, P.E., and Flick, D. "Twodimensional simulation of turbulent flow and transfer through stacked spheres", Int. J. Heat Mass Transf., 46(13), pp. 2459-2469 (2003).

10. Antohe, B.V. and Lage, J.L. "A general two-equation macroscopic turbulence model for incompressible flow in porous media", Int. J. Heat Mass Transf., 40(13), pp. 3013-3024 (1997).

11. Nakayama, A. and Kuwahara, F. "A macroscopic turbulence model for flow in a porous medium", J. Fluids Eng. Trans., ASME, 121(2), pp. 427-433 (1999).

12. Pedras, M.H.J. and De Lemos, M.J.S. "Macroscopic turbulence modeling for incompressible flow through undefromable porous media", Int. J. Heat Mass Transf., 44(6), pp. 1081-1093 (2001).

13. Carvalho, P.H.S. and de Lemos, M.J.S. "Role of porosity and solid-to-fluid thermal conductivity ratio on turbulent combined heat and mass transfer in a porous cavity", Int. J. Heat Mass Transf., 132, pp. 221-237 (2019).

14. Guo, B., Yu, A., Wright, B., and Zulli, P. "Comparison of several turbulence models applied to the simulation of gas flow in a packed bed", In 3rd International Conference on CFD in the Minerals and Process Industries, pp. 1-5 (Dec. 2003).

15. Nouri-Borujerdi, A. and Seyyed-Hashemi, M.H. "Numerical analysis of thermally developing turbulent flow in partially filled porous pipes", Sci. Iran., Trans. B, Mech. Eng., 22(3), p. 835 (2015).

16. Kazerooni, R.B. and Hannani, S.K. "Simulation of turbulent flow through porous media employing a v2f model", In AIP Conference Proceedings, 963(2), pp. $1257-1260$ (2007).

17. Kim, J.S. and Kang, P.K. "Anomalous transport through free-flow-porous media interface: Pore-scale simulation and predictive modeling", Adv. Water Resour., 135, p. 103467 (Jan. 2020).
18. de Lemos, M.J.S. and Assato, M. "Turbulence structure and heat transfer in a sudden expansion with a porous insert using linear and non-linear turbulence models", Int. J. Therm. Sci., 141, pp. 1-13 (July 2019).

19. Soulaine, C. and Quintard, M. "On the use of a DarcyForchheimer like model for a macro-scale description of turbulence in porous media and its application to structured packings", Int. J. Heat Mass Transf., 74, pp. 88-100 (2014).

20. Torabi, M., Torabi, M., Yazdi, M.E., and Peterson, G.P. "Fluid flow, heat transfer and entropy generation analyses of turbulent forced convection through isotropic porous media using RANS models", Int. J. Heat Mass Transf., 132, pp. 443-461 (2019).

21. Kundu, P., Kumar, V., and Mishra, I.M. "Numerical modeling of turbulent flow through isotropic porous media", Int. J. Heat Mass Transf., 75, pp. 40-57 (2014).

22. Chu, X., Yang, G., Pandey, S., and Weigand, B. "Direct numerical simulation of convective heat transfer in porous media", Int. J. Heat Mass Transf., 133, pp. 11-20 (2019).

23. Fu, J., Tang, Y., Li, J., Ma, Y., Chen, W., and Li, H. "Four kinds of the two-equation turbulence model's research on flow field simulation performance of DPF's porous media and swirl-type regeneration burner", Appl. Therm. Eng., 93, pp. 397-404 (Jan. 2016).

24. Khan, F.A. and Straatman, A.G. "Closure of a macroscopic turbulence and non-equilibrium turbulent heat and mass transfer model for a porous media comprised of randomly packed spheres", Int. J. Heat Mass Transf., 101, pp. 1003-1015 (2016).

25. Linsong, J., Hongsheng, L., Shaoyi, S., Maozhao, X., Dan, W., and Minli, B. "Pore-scale simulation of flow and turbulence characteristics in three-dimensional randomly packed beds", Powder Technol., 338, pp. 197-210 (2018).

26. Kuwahara, F., Kameyama, Y., Yamashita, S., and Nakayama, A. "Numerical modeling of turbulent flow in Porous media using a spatially periodic array", $J$. Porous Media, 1(1), pp. 47-55 (1998).

27. Kundu, P., Kumar, V., Hoarau, Y., and Mishra, I.M. "Numerical simulation and analysis of fluid flow hydrodynamics through a structured array of circular cylinders forming porous medium", Appl. Math. Model., 40(23-24), pp. 9848-9871 (2016).

28. Kim, S. "Numerical investigation on laminar pulsating flow through porous media", MS Thesis, Georgia Institute of Technology (2008).

29. Pathak, M.G. and Ghiaasiaan, S.M. "Convective heat transfer and thermal dispersion during laminar pulsating flow in porous media", Int. J. Therm. Sci., 50(4), pp. 440-448 (2011). 
30. Kardgar, A., Jafarian, A., and Arablu, M. "An Eulerian-Lagrangian model to study the operating mechanism of Stirling pulse tube refrigerators", Sci. Iran., Trans. B, Mech. Eng., 23(1), p. 277 (2016).

31. Rezaei, M., Jafarian, A., and Kardgar, A. "Numerical investigation of real gas effects on a two-stage pulse tube cryocooler performance", Int. J. Refrig., 82, pp. 106-118 (2017).

32. Nouri-Boroujerdi, A., Rabani, R., and Kermani, M.J. "Effect of porosity variation in determination of stagnant and dispersion thermal conductivities in the resin transfer molding process", Heat Transf. Res., 43(7), pp. 651-667 (2012).

33. Tu, S.W. and Ramaprian, B.R. "Fully developed periodic turbulent pipe flow. Part 1. Main experimental results and comparison with predictions", J. Fluid Mech., 137(137), pp. 31-58 (1983).

\section{Biographies}

Amin Kardgar received his BSc degree from University of Tehran in 2010 and MSc and PhD degrees from Tarbiat Modares University, Tehran, Iran in 2012 and 2017, respectively, all in Mechanical Engineering (Energy Conversion). He is currently a Professor of Mechanical Engineering at University of Mazandaran, Iran. His research interests include mathematical and numerical modeling, computational fluid dynamics, heat transfer in porous media, and turbulent combustion.

Ali Jafarian received his $\mathrm{BSc}$, MSc, and $\mathrm{PhD}$ degrees from Sharif University of Technology, Tehran, Iran in 2000, 2002, and 2008, respectively, all in Mechanical Engineering (Energy Conversion). He is currently a Professor of Mechanical Engineering at Tarbiat Modares University, Iran. 